\title{
Malignant Central Nervous System Neoplasm
}

National Cancer Institute

\section{Source}

National Cancer Institute. Malignant Central Nervous System Neoplasm. NCI Thesaurus.

Code C4627.

A primary or metastatic malignant neoplasm involving the brain or spinal cord.

Representative examples include anaplastic astrocytoma, glioblastoma, anaplastic

(malignant) meningioma, lymphoma, and metastatic carcinoma from another anatomic site. 